\title{
Análise crítica dos indicadores do estado nutricional de iodo em indivíduos e populações: uma revisão sistemática
}

\author{
Critical analysis of the indicators of the nutritional status of iodine \\ in individuals and populations: a systematic review
}

Aline Carare Candido (https://orcid.org/0000-0002-6259-4786) 1

Francilene Maria Azevedo (https://orcid.org/0000-0003-2162-5408) ${ }^{1}$

Mariana de Souza Macedo (https://orcid.org/0000-0003-4564-6334) ${ }^{1}$

Silvia Eloiza Priore (https://orcid.org/0000-0003-0656-1485) ${ }^{1}$

Sylvia do Carmo Castro Franceschini (https://orcid.org/0000-0001-7934-4858) ${ }^{1}$

${ }^{1}$ Programa de PósGraduação em Ciência da Nutrição, Departamento de Nutrição e Saúde, Universidade Federal de Viçosa. Av. Peter Henry Rolfs $\mathrm{s} / \mathrm{n}^{\circ}$, Ed. Centro de Ciências Biológicas II, Campus Universitário 36570.900 Viçosa

MG Brasil.

alinecarrare@gmail.com

\begin{abstract}
The scope of this article is to evaluate the potentialities of indicators of the nutritional status of iodine in individuals or populations. The review was based on PRISMA. The search for articles occurred in January 2019, in the Pubmed, Scopus and LILACS databases, using the key words: indicators AND nutritional status AND iodine. The selection followed the stages of excluding the duplicates, reading the titles, abstracts and analyses in full. The methodological quality of the studies was evaluated by the Downs and Black instrument. A total of 178 studies were identified and 20 were included. Urinary Iodine Concentration (UIC) was analyzed in $65 \%$ of the studies and was considered the best indicator to evaluate the nutritional status of iodine in the population. Thyroglobulin was determined in $20 \%$ of the studies and reflected the pre-existing state of iodine. Thyroid stimulating hormone was verified in $45 \%$ of the articles and was important for the surveillance of iodine deficiency among newborns. Only one study evaluated capillary iodine, useful for analyzing long-term dietary intake. In the evaluation of methodological quality, the lowest score was 12 and the highest 16, in 17 possible points. The use of UIC is recommended for the diagnosis of deficiency and excess of iodine in the population.
\end{abstract}

Key words Indicators, Iodine, Nutritional status
Resumo O objetivo deste artigo é avaliar as potencialidades dos indicadores do estado nutricional de iodo em indivíduos ou populações. A revisão foi baseada no PRISMA. A busca pelos artigos ocorreu em janeiro de 2019, nas bases Pubmed, Scopus e LILACS, utilizando a combinação "indicadores AND estado nutricional AND iodo". A seleção seguiu as etapas de exclusão dos duplicados, leitura de títulos e resumos e análise na integra. A qualidade metodológica dos estudos foi avaliada pelo instrumento de Downs e Black. Foram identificados 178 estudos e 20 foram incluídos. A Concentração Urinária de Iodo (CUI) foi analisada em $65 \%$ dos estudos e foi considerado o melhor indicador para avaliar o estado nutricional de iodo populacional. A tiroglobulina foi determinada em $20 \%$ dos estudos e refletiu o estado de iodo pregresso. O hormônio estimulante da tireoide foi verificado em $45 \%$ dos artigos e mostrou-se sensivel para a vigilância de deficiência de iodo em recém-nascidos. Apenas um estudo avaliou o iodo no cabelo, útil para analisar a ingestão dietética em longo prazo. Na avaliação da qualidade metodológica, a menor pontuação foi 12, e a maior 16, em 17 pontos possíveis. Para diagnóstico de deficiência e excesso de iodo na população, recomenda-se a CUI.

Palavras-chave Indicadores, Iodo, Estado nutricional 


\section{Introdução}

A deficiência de iodo acomete em todo o mundo aproximadamente 2 bilhões de pessoas, por isso é considerada um problema de saúde pública. Provoca efeitos adversos à saúde, denominados distúrbios por deficiência de iodo (DDI), devido à produção insuficiente de hormônios tireoidianos $^{1}$.

As consequências para a saúde associadas à deficiência de iodo atingem todos os públicos, em especial as crianças durante o desenvolvimento intrauterino e no primeiro ano de vida, fases nas quais ocorre o neurodesenvolvimento, que é dependente de hormônios tireoidianos. Essa deficiência pode provocar também danos econômicos e sociais, por isso o seu controle é uma questão crítica de desenvolvimento e deve ser ação prioritária dos governos ${ }^{2}$.

A Organização Mundial de Saúde (OMS) recomenda a iodação universal do sal em todas as áreas deficientes, por ser um método simples, eficaz e de baixo custo para assegurar a ingestão adequada de iodo por toda a população ${ }^{3}$.

O programa de iodação do sal, assim como o estado nutricional de iodo da população, exige monitoramento. Para isso são utilizados indicadores que dimensionam se estratégias para controle da deficiência de iodo se darão no curto, médio ou longo prazos ${ }^{4}$.

Quatro indicadores são os mais utilizados em estudos epidemiológicos: a concentração urinária de iodo, a tiroglobulina, o hormônio estimulante da tireoide (TSH) e o volume tireoidiano. Recentemente, os estudos vêm analisando o conteúdo de iodo no cabelo ${ }^{5,6}$.

$\mathrm{Na}$ literatura existem questionamentos sobre os indicadores do estado nutricional de iodo, em nível populacional ou individual, de acordo com faixa etária e estado fisiológico, por isso nosso objetivo foi avaliar as potencialidades dos indicadores do estado nutricional de iodo em indivíduos ou populações analisados nos estudos revisados, bem como suas aplicações, diante da classificação realizada pela OMS.

\section{Metodologia}

Trata-se de uma revisão sistemática, baseada nas recomendações do Preferred Reporting Items for Systematic Reviews (PRISMA) ${ }^{7}$.

A OMS utiliza os indicadores concentração urinária de iodo, tiroglobulina, hormônio estimulante da tireoide e volume tireoidiano para avaliar o impacto e a sustentabilidade da política de iodação do sal, verificando indiretamente o estado nutricional de iodo populacional. No entanto, cada indicador é específico ou mais sensível para avaliar determinada faixa etária. Por isso é importante conhecer os indicadores para selecionar o mais adequado de acordo com o público estudado.

Nossa pergunta norteadora foi: "quais os indicadores mais utilizados para avaliar o estado nutricional de iodo em indivíduos e populações?" A busca pelos artigos ocorreu em janeiro de 2019, sem delimitação de datas.

As bases de dados consultadas foram: $\mathrm{Pu}-$ blisher Medline (Pubmed), Literatura Latino -Americana e do Caribe em Ciências da Saúde (LILACS) e Scopus. Os descritores em ciência da saúde (DeCS) fornecem um vocabulário estruturado pela Biblioteca Regional de Medicina (BIREME) para indexação de artigos. Os descritores utilizados foram "estado nutricional" e "iodo". Como o objetivo do artigo foi identificar os indicadores que avaliam o estado nutricional de dessa substância, optamos por incluir a palavra-chave "indicadores" na busca. Logo, nas bases de dados foi utilizada a combinação "indicadores" AND "estado nutricional" AND "iodo" em inglês, português e espanhol. Utilizou-se o filtro humano.

Foram incluídos todos os artigos que detalharam os indicadores do estado nutricional de iodo. Excluíram-se revisões sistemáticas, artigos referentes a outros micronutrientes que não incluíram o iodo, estudos que realizaram suplementação/fortificação de alimentos com iodo e que abordaram outros temas, como câncer e diabetes.

A seleção dos artigos foi executada por dois pesquisadores e de maneira independente. Quando a opinião era distinta em relação aos estudos, entravam em acordo para obter a melhor decisão. Realizou-se primeiro a leitura dos títulos, depois dos resumos e por fim dos artigos completos; caso não atendessem ao critério de inclusão, os estudos eram eliminados.

Após a seleção, foram registrados em uma planilha no Excel todos os dados dos artigos, como autores, método utilizado para diagnosticar o estado nutricional de iodo, principais resultados e conclusões sobre os indicadores utilizados.

A qualidade metodológica dos estudos foi avaliada pelo instrumento proposto por Downs e Black ${ }^{8}$, que contém quatro categorias: relato do estudo (clareza), validade externa (representati- 
vidade), validade interna (vieses e fatores confundidores) e poder estatístico do estudo. Foram utilizados 17 das 27 perguntas, pois dez se referiam a estudos experimentais. As respostas foram pontuadas com o valor "1" (quando o critério que caracterizasse qualidade estivesse presente) ou "0" (quando o critério estivesse ausente). O sistema de classificação adaptado indica a qualidade do estudo com escores de zero (pior qualidade) a 17 (melhor qualidade).

\section{Resultados}

A busca resultou em 178 artigos. Após eliminar as duplicidades por bases e entre as bases, restaram 108. Depois da leitura dos títulos, resumos e artigos na íntegra, 20 foram incluídos (Figura 1).

Os anos dos estudos variaram de $1992^{9}$ a $2016^{2,4,6}$, sendo $70 \%$ de delineamento transversal. O tamanho amostral variou de $22^{10}$ a $140 \mathrm{mil}^{11}$ indivíduos, e o principal indicador utilizado em $65 \%$ dos estudos foi a concentração urinária de iodo. A tiroglobulina foi avaliada em $20 \%$ dos estudos, o TSH em $45 \%$ e o volume tireoidiano em $25 \%$. Todos os públicos, com diferentes faixas etárias, foram contemplados nos artigos (Quadro 1).

O Quadro 2 descreve os principais resultados dos estudos incluídos nesta revisão, detalhando a referência utilizada para diagnosticar o estado nutricional de iodo, os principais resultados detectados e as conclusões a respeito dos indicadores utilizados.

A faixa para classificação da concentração urinária de iodo (CUI) populacional em "adequada", "leve", "moderada" e "grave", proposta em 1992, foi modificada em 2007. Incluiu-se uma classificação específica para gestantes e foram acrescentados os pontos de corte "acima do

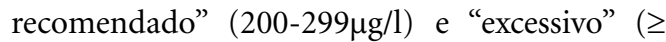
$300 \mu \mathrm{g} / \mathrm{l})^{1}$, conforme a Quadro 3. Para os demais indicadores, a classificação manteve-se a mesma com o decorrer dos anos.

Na referência de 1992 e 1994, a CUI acima de $100 \mu \mathrm{g} / \mathrm{l}$ indicava ingestão dietética de iodo adequada $^{26,27}$. Em 2007, a OMS ${ }^{1}$ propôs uma nova classificação para a CUI, tornando possível o diagnóstico de excesso de iodo.

$\mathrm{Na}$ avaliação dos estudos segundo Downs e Black $^{8}$, a menor pontuação foi $12(10 \%)$, e a maior $16(35 \%)$, indicando a boa qualidade metodológica e que os achados são confiáveis e seguros. Os critérios melhor avaliados foram o relato de estudo (desfechos principais medidos descritos na metodologia, principais achados descritos, variabilidade randômica dos dados para os desfechos e valores reais de probabilidade reportados integralmente), a validade externa (representatividade da amostra, tempo de seguimento igual para todos os indivíduos e desfechos com medidas confiáveis) e interna (indivíduos recrutados na mesma população e no mesmo período de tempo). No entanto, de todos os estudos incluídos, nenhum apresentou poder estatístico (Tabela 1).

\section{Discussão}

Os indicadores descrevem uma situação ou rastreiam mudanças ao longo do tempo, podendo ser quantitativos ou qualitativos. Para avaliar o estado nutricional de iodo, os indicadores são divididos em três grupos: de processo, impacto e sustentabilidade ${ }^{1}$.

\section{Indicadores do processo}

Avaliam o teor de iodo no sal e fiscalizam se a indústria alimentícia está respeitando a faixa recomendada. Para monitorar os programas de controle dos distúrbios por deficiência de iodo (DDI), pode-se verificar o teor de iodo no sal, os fatores que podem afetar sua estabilidade, além de analisar sua disponibilidade no domicílio, os hábitos alimentares e as práticas culinárias da população ${ }^{1}$.

Em estudo realizado na Nova Zelândia, 51\% dos escolares não consumiam sal iodado e a CUI indicou leve deficiência ${ }^{16}$. Já na Espanha, Santiago e colaboradores ${ }^{11}$ observaram que $60,1 \%$ dos escolares consumiam sal iodado, no entanto, detectaram baixos valores de CUI, que caracterizaram deficiência moderada. A maior prevalência de deficiência de iodo foi verificada nas crianças residentes na zona rural, o que pode ser atribuído ao fato de elas só consumirem sal iodado na escola.

Apenas um estudo (5\%) avaliou o teor de iodo no sal e os autores observaram variação de $24,5 \mathrm{ppm}$ a $39,6 \mathrm{ppm}$, dentro da faixa de $15-40$ ppm recomendada no Egito atualmente ${ }^{2}$. É importante destacar que a OMS ${ }^{1}$ determina que a concentração de iodo no sal em todo o mundo esteja dentro do intervalo de $20-40 \mathrm{ppm}$, para atender à recomendação de $150 \mu \mathrm{g} / \mathrm{dia}$ de iodo para os indivíduos. Mas, de acordo com as características de cada local, essa faixa pode ser alterada.

Os estudos demostraram que quando era consumido sal não iodado, a CUI era menor, por 


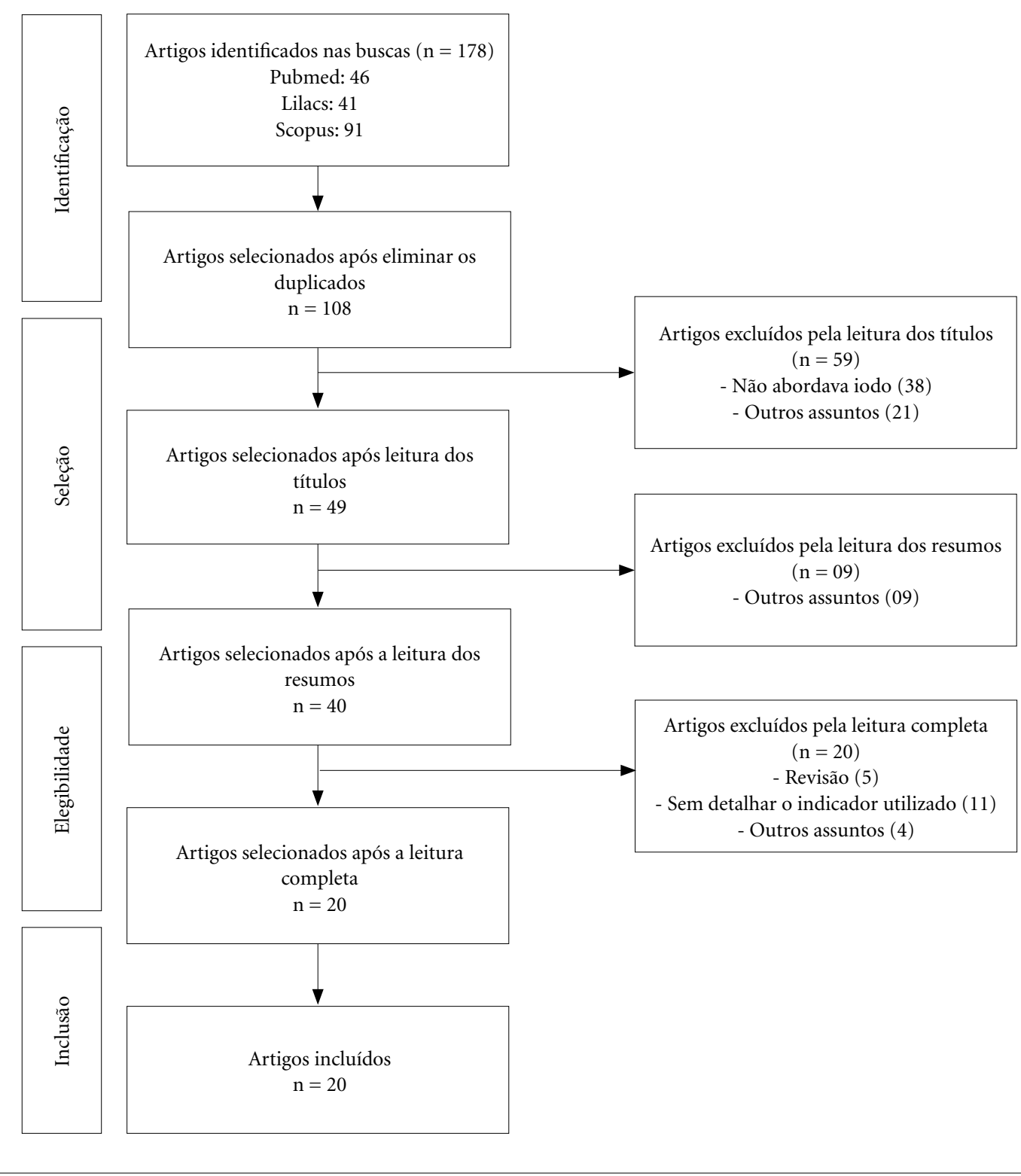

Figura 1. Fluxograma do processo de identificação e seleção dos artigos incluídos.

Fonte: PRISMA ${ }^{7}$

isso é um bom indicador do consumo de iodo proveniente do sal. Para monitorar se a indústria alimentícia está respeitando a faixa de iodação, que difere de um país para outro, recomenda-se a avaliação laboratorial do teor de iodo no sal de consumo.

\section{Indicadores de impacto}

Avaliam o estado nutricional do iodo e o impacto da iodação do sal na população. Nesse caso, a OMS ${ }^{1}$ recomenda utilizar a CUI, o volume tireoidiano por meio da palpação ou ultrassonografia para detectar o bócio e alguns marcadores sorológicos para analisar a função tireoidiana, como os hormônios estimulantes da tireoide (TSH), a tireoglobulina ( $\mathrm{Tg}$ ), a triiodotironina (T3) e a tiroxina (T4).

Cerca de $90 \%$ do iodo absorvido é excretado na urina em 24 horas, podendo ser expresso em concentração (CUI) ou em quantidade diária excretada (mg/24h). A CUI reflete a ingestão re- 
Quadro 1. Descrição dos estudos selecionados para a revisão sistemática.

\begin{tabular}{|c|c|c|c|c|}
\hline Autores/ano & Local & Delineamento & $\begin{array}{c}\text { Tamanho amostral } \\
(\mathbf{n})\end{array}$ & Indicador \\
\hline Brug et al., $1992^{9}$ & Holanda & Transversal & 812 adultos & $\begin{array}{l}\text { CUI, relação iodo/creatinina em } \\
\text { amostras de urina de } 24 \text { horas, } \\
\text { iodúria de } 24 \text { horas/quilograma de } \\
\text { peso corporal }\end{array}$ \\
\hline $\begin{array}{l}\text { Cherinet, Kelbess } \\
2000^{12}\end{array}$ & Etiópia & Transversal & 2.485 escolares & $\begin{array}{l}\text { Iodúria, concentração de iodo na água } \\
\text { e contaminação bacteriana da água }\end{array}$ \\
\hline Eltom et. al, $2000^{13}$ & Suécia & Longitudinal & $\begin{array}{l}48 \text { gestantes e grupo } \\
\text { controle com } 44 \\
\text { mulheres }\end{array}$ & CUI, Tg, TSH, T3 e FT4 \\
\hline Costante et. al, $2002^{14}$ & Itália & Longitudinal & 13.984 escolares & CUI, volume da tireoide, TSH e T4 \\
\hline Delange et al., $2002^{15}$ & $\begin{array}{l}17 \text { países } \\
\text { de quatro } \\
\text { continentes }\end{array}$ & Transversal & $\begin{array}{l}55.892(35.661 \\
\text { escolares e } 20.231 \\
\text { adultos) }\end{array}$ & CUI \\
\hline $\begin{array}{l}\text { Skeaff, Thomson, } \\
\text { Gibson, } 2002^{16}\end{array}$ & Nova Zelândia & Transversal & 300 escolares & $\begin{array}{l}\text { CUI, volume da tireoide e consumo } \\
\text { de sal iodado }\end{array}$ \\
\hline Simsek et. al, $2003^{17}$ & Turquia & Transversal & 727 escolares & $\begin{array}{l}\text { Tg, T4, FT4, TSH, volume da tireoide } \\
\text { e percentil da altura }\end{array}$ \\
\hline Soldin et al., $2004^{18}$ & Suécia & Longitudinal & $\begin{array}{l}47 \text { gestantes na } 12^{\mathrm{a}}, \\
22^{\mathrm{a}} \text { e } 32^{\mathrm{a}} \text { semana de } \\
\text { gestação e com um } \\
\text { ano pós-parto }\end{array}$ & T4, TSH, T3, FT4 e Tg \\
\hline Santiago et al., $2005^{11}$ & Espanha & Transversal & 140.000 escolares & CUI e consumo de sal iodado \\
\hline $\begin{array}{l}\text { Soldin, Tractenberg, } \\
\text { Pezzullo, } 2005^{19}\end{array}$ & $\begin{array}{l}\text { Estados } \\
\text { Unidos }\end{array}$ & Transversal & $\begin{array}{l}7.628 \text { indivíduos } \\
\text { com idade entre } 15 \\
\text { e } 44 \text { anos }\end{array}$ & Iodúria, TSH e T4 \\
\hline Ategbo et al., $2008^{20}$ & Índia & Transversal & $\begin{array}{l}\text { Gestantes e escolares } \\
\text { de } 1.200 \text { domicílios }\end{array}$ & $\begin{array}{l}\text { CUI e consumo domiciliar de sal } \\
\text { iodado }\end{array}$ \\
\hline $\begin{array}{l}\text { Dorey, Zimmermann, } \\
2008^{21}\end{array}$ & Suíça & Transversal & 634 recém-nascidos & CUI e TSH \\
\hline $\begin{array}{l}\text { Barona-Vilar, } \\
\text { Montoro, } 2008^{22}\end{array}$ & Espanha & Transversal & $\begin{array}{l}91.853 \text { recém- } \\
\text { nascidos }\end{array}$ & TSH \\
\hline $\begin{array}{l}\text { Elahi, Rizvi, Nagra, } \\
2009^{23}\end{array}$ & Índia & Transversal & 254 gestantes & CUI \\
\hline Konig et al., $2011^{10}$ & Suíça & Longitudinal & $\begin{array}{l}22 \text { mulheres entre } \\
52 \text { e } 77 \text { anos }\end{array}$ & CUI \\
\hline Prejac et al., $2014^{5}$ & Croácia & Transversal & $\begin{array}{l}870 \text { adultos }(270 \\
\text { homens e } 600 \\
\text { mulheres })\end{array}$ & Iodo no cabelo \\
\hline Du et al., $2014^{24}$ & China & Transversal & 2.147 Adultos & $\begin{array}{l}\text { Concentração de iodo na água, CUI, } \\
\text { TSH, T3, T4 }\end{array}$ \\
\hline Li et al., $2016^{6}$ & China & Longitudinal & $\begin{array}{l}222 \text { gestantes e } 827 \\
\text { puérperas }\end{array}$ & Iodúria, creatinina, Tg e iodo sérico. \\
\hline Yamamah et al., $2016^{2}$ & Egito & Transversal & 1.046 escolares & $\begin{array}{l}\text { Volume da tireoide, CUI e consumo } \\
\text { de sal iodado }\end{array}$ \\
\hline $\begin{array}{l}\text { Zoysa, Hettiarachchi, } \\
\text { Liyanage, } 2016^{4}\end{array}$ & Sri Lanka & Prospectivo & 425 gestantes & CUI, TSH e volume da tireoide \\
\hline
\end{tabular}

*(CUI): concentração urinária de iodo; (Tg) tiroglobulina; (TSH) hormônio estimulante da tireoide; (T3) triidotironina; (T4) tiroxina.

cente de iodo, por isso é considerada a principal referência para avaliação da população e monitoramento de intervenções ${ }^{10}$.
A CUI foi considerada o melhor indicador para avaliar a deficiência de iodo em nível populacional ${ }^{11,19,20}$. No entanto, para a avaliação indivi- 
Quadro 2. Principais resultados dos estudos.

\begin{tabular}{|c|c|c|c|}
\hline Autores & Referência utilizada & Resultados & Principais conclusões \\
\hline Brug et al., $1992^{9}$ & HETZEL $(1989)^{25}$ & $\begin{array}{l}35 \% \text { dos homens tinham } 1 / 24 \text { h baixa, } \\
36 \% \text { tinham um I/Cr baixa e } 18 \% \text { tinham } \\
\text { um I/kg baixo. Entre as mulheres, esses } \\
\text { percentuais foram } 64 \% \text {, } 33 \% \text { e } 26 \% \text {. }\end{array}$ & $\begin{array}{l}\text { I/Cr e I/kg são melhores indicadores } \\
\text { para o estado nutricional de iodo, } \\
\text { pois levam em consideração a } \\
\text { estrutura e composição corporal. }\end{array}$ \\
\hline $\begin{array}{l}\text { Cherinet, Kelbess, } \\
2000^{12}\end{array}$ & $\begin{array}{l}\text { WHO, UNICEF, } \\
\text { ICCIDD }(1994)^{26}\end{array}$ & $\begin{array}{l}53,3 \% \text { dos escolares apresentaram bócio, } \\
\text { sendo a prevalência maior em crianças que } \\
\text { vivem em aldeias com água contaminada e } \\
\text { com baixo conteúdo de iodo. }\end{array}$ & $\begin{array}{l}\text { O melhor indicador para avaliar } \\
\text { o estado nutricional de iodo na } \\
\text { população é a iodúria, pois reflete a } \\
\text { ingestão de iodo atual. }\end{array}$ \\
\hline Eltom et al, $2000^{13}$ & $\begin{array}{l}\text { WHO, UNICEF, } \\
\text { ICCIDD }(1994)^{26}\end{array}$ & $\begin{array}{l}\text { A Tg foi maior no terceiro trimestre de } \\
\text { gestação e inferior para gestantes suecas } \\
\text { quando comparadas às sudanesas. A CUI } \\
\text { foi maior nas gestantes suecas. }\end{array}$ & $\begin{array}{l}\text { A Tg é um indicador mais sensível } \\
\text { para deficiência de iodo do que o } \\
\text { TSH durante a gravidez. }\end{array}$ \\
\hline $\begin{array}{l}\text { Costante et al., } \\
2002^{14}\end{array}$ & $\begin{array}{l}\text { WHO, UNICEF, } \\
\text { ICCIDD }(1994)^{26}\end{array}$ & $\begin{array}{l}\text { A prevalência de bócio nos meninos foi de } \\
28,2 \% \text {, e nas meninas de } 42,8 \% \text {. Em } 14,8 \% \\
\text { dos recém-nascidos do interior os níveis de } \\
\text { TSH foram }>5 \mu \mathrm{U} / \mathrm{ml} \text {. A iodúria média dos } \\
\text { escolares do interior foi de } 53,8 \pm 43,4 \mu \mathrm{g} / \mathrm{l} \text {, } \\
\text { e no nível do mar foi de } 89,6 \pm 59,8 \mu \mathrm{g} / \mathrm{l} \text {. }\end{array}$ & $\begin{array}{l}\text { TSH neonatal é sensível para a } \\
\text { vigilância da deficiência de iodo. } \\
\text { E a iodúria é o indicador útil para } \\
\text { avaliar a gravidade de deficiência de } \\
\text { iodo. }\end{array}$ \\
\hline $\begin{array}{l}\text { Delange et al., } \\
2002^{15}\end{array}$ & $\begin{array}{l}\text { WHO, UNICEF, } \\
\text { ICCIDD }(1994)^{26}\end{array}$ & $\begin{array}{l}\text { As concentrações de iodo variaram de } 111 \\
\text { a } 540 \mu \mathrm{g} / \mathrm{l} \text { nos adultos. A CUI foi de } 100 \\
\text { a } 199 \mu \mathrm{g} \text { em } 48 \% \text { dos escolares, indicando } \\
\text { suficiência iódica. }\end{array}$ & $\begin{array}{l}\text { A CUI varia durante o dia e de um } \\
\text { dia para outro, por isso uma única } \\
\text { medição não é representativa do } \\
\text { estado nutricional individual. }\end{array}$ \\
\hline $\begin{array}{l}\text { Skeaff, Thomson, } \\
\text { Gibson, } 2002^{16}\end{array}$ & $\begin{array}{l}\text { WHO, UNICEF, } \\
\text { ICCIDD }(1994)^{26} ; \\
\text { WHO, ICCIDD } \\
(1997)^{28}\end{array}$ & $\begin{array}{l}51 \% \text { dos escolares não utilizavam sal } \\
\text { iodado, } 3,6 \% \text { tiveram níveis de CUI } \\
\text { inferiores a } 2 \mathrm{mg} / \mathrm{dl} \text { e } 79,7 \% \text { superiores } \\
\text { a } 10 \mathrm{mg} / \mathrm{dl} \text {, indicando deficiência leve de } \\
\text { iodo; } 3 \% \text { apresentaram volume da tiroide } \\
\text { anormal. }\end{array}$ & $\begin{array}{l}\text { O volume da tireoide é variável na } \\
\text { infância. E a limitação para utilizar } \\
\text { a ultrassonografia para medir o } \\
\text { volume da tireoide em crianças se } \\
\text { deve a não haver pontos de corte } \\
\text { para a classificação. }\end{array}$ \\
\hline Simsek et al., $2003^{17}$ & $\begin{array}{l}\text { WHO, ICCIDD } \\
(1997)^{28}\end{array}$ & $\begin{array}{l}\text { A CUI foi grave em } 38 \% \text { dos escolares, } \\
\text { moderada em } 21 \% \text { e leve em } 16 \% \text {. O } \\
\text { percentil da altura para os escolares com } \\
\text { deficiência grave foi de } 23 \mathrm{~cm} \text {, moderada } \\
\text { foi de } 30 \mathrm{~cm} \text { e leve de } 44 \mathrm{~cm} \text {. A Tg média } \\
\text { foi de } 22,1 \mathrm{ng} / \mathrm{ml} \text {. Os níveis de FT4 nos } \\
\text { escolares com deficiência de iodo grave foi } \\
1,04 \mathrm{ng} / \mathrm{dl}, \mathrm{moderada} \text { foi de } 1,14 \mathrm{ng} / \mathrm{dl} \mathrm{e} \\
\text { leve de } 1,19 \mathrm{ng} / \mathrm{dl} \text {. }\end{array}$ & $\begin{array}{l}\text { O percentil da altura e a Tg podem } \\
\text { ser indicadores sensíveis para } \\
\text { demonstrar o efeito crônico da } \\
\text { deficiência de iodo em crianças. } \\
\text { FT4 e Tg são mais sensíveis para } \\
\text { classificar a gravidade da deficiência } \\
\text { de iodo. }\end{array}$ \\
\hline Soldin et al., $2004^{18}$ & $\begin{array}{l}\text { SOUKHOVA, } \\
\text { SOLDIN, SOLDIN } \\
(2004)^{29}\end{array}$ & $\begin{array}{l}\text { As concentrações de TSH, T3 e T4 são } \\
\text { maiores durante a gravidez do que no pós- } \\
\text { parto. T4 permaneceu estável durante a } \\
\text { gravidez e T3 aumentou a cada trimestre. } \\
\text { FT4 foi menor durante a gravidez do que } \\
\text { no período pós-parto. A Tg foi mais alta } \\
\text { durante o terceiro trimestre. }\end{array}$ & $\begin{array}{l}\text { T3, FT4, TSH e Tg tendem a mudar } \\
\text { ao longo da gestação, por isso não } \\
\text { são bons indicadores de deficiência } \\
\text { de iodo. }\end{array}$ \\
\hline
\end{tabular}

dual do estado nutricional de iodo, a CUI não é indicada, devido à variação durante o dia e de um dia para outro. Além disso, não está claro quantas repetições de urina são necessárias para que os resultados sejam precisos e se as amostras de urina devem ser casuais ou de 24 horas. Porém, esse indicador é útil quando utilizado em estudo populacional, transversal e epidemiológico ${ }^{10,15}$.

Brug e colaboradores ${ }^{9}$ avaliaram, em 1992, a iodúria de 24 horas (I/24h), as relações iodo/ creatinina (I/Cr) e iodo/quilo corporal (I/kg) em adultos e concluíram que I/Cr e I/kg são indica- 
Quadro 2. Principais resultados dos estudos.

\begin{tabular}{|c|c|c|c|}
\hline Autores & Referência utilizada & Resultados & Principais conclusões \\
\hline $\begin{array}{l}\text { Santiago et al., } \\
2005^{11}\end{array}$ & $\begin{array}{l}\text { WHO, UNICEF, } \\
\text { ICCIDD }(1994)^{26}\end{array}$ & $\begin{array}{l}\text { 60,1\% dos escolares consomem sal iodado. } \\
\text { A CUI foi de } 102,9 \mu \mathrm{g} \text { (adequada). Porém } \\
\text { na zona rural e em crianças que não } \\
\text { consomem sal iodado a CUI foi inferior } \\
\text { a } 100 \mu \mathrm{g} \text { (moderada). Em } 19,2 \% \text { dos } \\
\text { escolares a CUI foi inferior a } 50 \mu \mathrm{g} \text { (grave). }\end{array}$ & $\begin{array}{l}\text { A CUI é o melhor indicador para } \\
\text { avaliar a deficiência de iodo e o } \\
\text { progresso da iodação do sal em } \\
\text { escolares, pois reflete a ingestão atual } \\
\text { de iodo. }\end{array}$ \\
\hline $\begin{array}{l}\text { Soldin, Tractenberg, } \\
\text { Pezzullo, } 2005^{19}\end{array}$ & $\begin{array}{l}\text { WHO, UNICEF, } \\
\text { ICCIDD }(1994)^{26}\end{array}$ & $\begin{array}{l}\text { A CUI foi menor em mulheres em } \\
\text { comparação aos homens da mesma faixa } \\
\text { etária. T4 foi maior nas mulheres em } \\
\text { comparação aos homens. As medianas } \\
\text { e médias da CUI e T4 foram maiores } \\
\text { nas gestantes. Já os níveis de TSH foram } \\
\text { menores em gestantes do que em mulheres } \\
\text { não grávidas. }\end{array}$ & $\begin{array}{l}\text { T4 e TSH são indicadores indiretos } \\
\text { de deficiência de iodo. O TSH } \\
\text { é um bom biomarcador apenas } \\
\text { para triagem de tireoide neonatal. } \\
\text { A CUI é indicada para avaliar a } \\
\text { deficiência de iodo em nível apenas } \\
\text { populacional. }\end{array}$ \\
\hline $\begin{array}{l}\text { Ategbo et al., } \\
2008^{(20)}\end{array}$ & WHO $(2007)^{(1)}$ & $\begin{array}{l}\text { O teor de iodo salino foi } \geq 15 \mathrm{ppm} \text { em } \\
41,9 \% \text { dos domicílios e } 23 \% \text { usam sal não } \\
\text { iodado. A CUI foi de } 139 \mu \mathrm{g} \text { em crianças e } \\
127 \mu \mathrm{g} \text { em gestantes. } 20,9 \% \text { das gestantes } \\
\text { tinham grave deficiência de iodo e } 43,3 \% \\
\text { moderada. Nos domicílios que usam sal } \\
\text { não iodado, as medianas da CUI foram } \\
\text { menores, } 96 \mu \mathrm{g} \text { em crianças e } 100 \mu \mathrm{g} \text { em } \\
\text { gestantes. }\end{array}$ & $\begin{array}{l}\text { A CUI reflete a ingestão atual de } \\
\text { iodo, por isso deve ser considerada } \\
\text { o melhor indicador para avaliar } \\
\text { deficiência de iodo. }\end{array}$ \\
\hline $\begin{array}{l}\text { Dorey, } \\
\text { Zimmermann, } \\
2008^{21}\end{array}$ & WHO $(2007)^{1}$ & $\begin{array}{l}\text { A CUI foi de } 77 \mu \text { g nos recém-nascidos, } \\
\text { sendo que nenhum apresentou TSH } \\
\text { elevado; } 92 \% \text { estavam em amamentação } \\
\text { exclusiva. A média de creatinina foi de } 478 \\
\mu g \text { (alta). }\end{array}$ & $\begin{array}{l}\text { A creatinina variou de duas a três } \\
\text { vezes ao longo dos dias, sugerindo } \\
\text { que ela não é útil para avaliar } \\
\text { a excreção de iodo na primeira } \\
\text { semana de vida. Já o TSH é um bom } \\
\text { indicador do estado de iodo em } \\
\text { recém-nascidos. }\end{array}$ \\
\hline $\begin{array}{l}\text { Barona-Vilar, } \\
\text { Montoro, } 2008^{22}\end{array}$ & WHO $(2007)^{1}$ & $\begin{array}{l}\text { Nos recém-nascidos com menos de dois } \\
\text { anos de vida, a mediana do TSH foi de 2,19 } \\
\text { mU/L. A prevalência de TSH > } 5 \text { mU/L } \\
\text { foi maior ( } 4 \% \text { versus } 1,9 \% \text { em crianças } \\
\text { nascidas em maternidades que usaram } \\
\text { antissépticos de iodo. }\end{array}$ & $\begin{array}{l}\text { O TSH é um bom indicador para } \\
\text { monitorar o estado nutricional de } \\
\text { iodo populacional, mas é necessário } \\
\text { eliminar o uso perinatal de } \\
\text { antissépticos iodado, pois este eleva } \\
\text { o valor do TSH. }\end{array}$ \\
\hline $\begin{array}{l}\text { Elahi, Rizvi Nagra, } \\
2009^{23}\end{array}$ & WHO $(2007)^{1}$ & $\begin{array}{l}\text { A iodúria variou de } 34 \mathrm{~g} / \mathrm{l} \text { a } 142 \mathrm{~g} / \mathrm{l} \text { e a } \\
\text { mediana foi de } 67 \mathrm{~g} / \mathrm{l} .68,8 \% \text { das gestantes } \\
\text { estavam com deficiência leve de iodo e } \\
24,8 \% \text { com moderada. } 31,5 \% \text { das gestantes } \\
\text { apresentaram bócio levemente visível. } \\
34,2 \% \text { estavam em uso de sal iodado. }\end{array}$ & $\begin{array}{l}\text { O aumento no volume da tireoide } \\
\text { é um indicador indireto de } \\
\text { deficiência de iodo. Já a iodúria é } \\
\text { um bom indicador, pois mostra } \\
\text { a disponibilidade de iodo antes e } \\
\text { durante a gestação. }\end{array}$ \\
\hline
\end{tabular}

dores melhores para o estado nutricional de iodo, quando comparados a $\mathrm{I} / 24 \mathrm{~h}$, pois levam em consideração a estrutura e a composição corporal.

Porém, no estudo de Dorey e Zimmermannn ${ }^{21}$, realizado com recém-nascidos em 2008, a creatinina variou de duas a três vezes ao longo do dia, sugerindo que ela não é útil para avaliar a excreção de iodo na primeira semana de vida. No estudo realizado por Li e colaboradores ${ }^{6}$ em 2016 com gestantes e puérperas, foi analisado o iodo sérico e a relação iodúria/creatinina (UI/Cr). A área sob a curva da UI/Cr foi de 0,92 para avaliar deficiência de iodo e de 0,78 para iodo sérico e para excesso de iodo, e de 0,75 para UI/Cr e 0,82 
Quadro 2. Principais resultados dos estudos.

\begin{tabular}{|c|c|c|c|}
\hline Autores & Referência utilizada & Resultados & Principais conclusões \\
\hline Konig et al., $2011^{10}$ & $\begin{array}{l}\text { RARTELS, } \\
\text { BOHMER }(1971)^{30} ; \\
\text { PINO et al. }(1996)^{31} \\
\text { EUSER et al, } \\
(2008)^{32}\end{array}$ & $\begin{array}{l}\text { A CV foi comparada entre a UIE medida e } \\
\text { a estimada. A CV foi mais alta para a UIC } \\
\text { medida ( } 38 \%) \text { do que para a estimada em } \\
24 \text { horas }(33 \%) \text {. Foram necessárias dez } \\
\text { amostras de urina para avaliar o estado de } \\
\text { iodo individual com } 20 \% \text { de precisão. }\end{array}$ & $\begin{array}{l}\text { A CUI é um bom indicador do } \\
\text { estado de iodo nas populações, } \\
\text { porém se for utilizada para } \\
\text { avaliar indivíduos, não está claro } \\
\text { quantas repetições de urina seriam } \\
\text { necessárias e se deveriam ser } \\
\text { amostras casuais ou de } 24 \text { horas. }\end{array}$ \\
\hline Prejac et al., $2014^{5}$ & IAEA $(1980)^{33}$ & $\begin{array}{l}\text { A mediana da concentração de iodo do } \\
\text { cabelo entre homens e mulheres foi de } \\
0,499 \mu \mathrm{gg}^{-1} \text {, sugerindo nível nutricional } \\
\text { inadequado de ingestão de iodo. }\end{array}$ & $\begin{array}{l}\text { O iodo do cabelo pode ser usado } \\
\text { como um indicador valioso e } \\
\text { robusto da ingestão dietética a longo } \\
\text { prazo. }\end{array}$ \\
\hline Du et al., $2014^{24}$ & WHO $(2007)^{1}$ & $\begin{array}{l}\text { Na correlação de Spearman a CUI foi } \\
\text { positivamente relacionada ao TSH }(\mathrm{r}= \\
0,414, \mathrm{p}=0) . \text { Na regressão linear múltipla, } \\
\text { após ajuste para sexo e idade, o TSH } \\
\text { correlacionou-se com o excesso de ingestão } \\
\text { de iodo }(\mathrm{b}=1.764, \mathrm{p}=0.001) \text { e com } \\
\text { ingestão deficiente }(\mathrm{b}=21.219, \mathrm{p}=0,028) \text {. }\end{array}$ & $\begin{array}{l}\text { A mediana do TSH pode ser } \\
\text { considerada um bom indicador } \\
\text { para o monitoramento do estado } \\
\text { nutricional de iodo da população } \\
\text { adulta em excesso e deficiência. }\end{array}$ \\
\hline Li et al., $2016^{6}$ & WHO $(2007)^{1}$ & $\begin{array}{l}\text { A iodúria diminuiu de } 183,6 \mu \mathrm{g} / \mathrm{l} \text { para } \\
104,2 \mu \mathrm{g} / \mathrm{l} \text { durante a gestação. O iodo sérico } \\
\text { foi reduzido de } 106,5 \mu \mathrm{g} / \mathrm{l} \text { na } 20^{\mathrm{a}} \text { semana } \\
\text { para } 84,7 \mu \mathrm{g} / \mathrm{l} \text { na } 36^{\mathrm{a}} \text { semana. A área sob a } \\
\text { curva da UI/Cr foi de } 0,92 \text { para diagnóstico } \\
\text { de deficiência de iodo e } 0,78 \text { para iodo } \\
\text { sérico. E para diagnóstico de excesso de } \\
\text { iodo foi de } 0,82 \text { para o iodo sérico e } 0,75 \\
\text { para UI/Cr. }\end{array}$ & $\begin{array}{l}\text { A iodúria é um excelente indicador } \\
\text { do consumo recente de iodo. A UI/ } \\
\text { Cr reflete melhor a excreção de } \\
\text { iodo em } 24 \text { horas e os níveis de } \\
\text { iodo circulante. Para diagnóstico de } \\
\text { deficiência de iodo, indica-se UI/Cr, } \\
\text { e para diagnósticos de excessos de } \\
\text { iodo, recomenda-se o iodo sérico. }\end{array}$ \\
\hline $\begin{array}{l}\text { Yamamah et al., } \\
2016^{2}\end{array}$ & WHO $(2007)^{1}$ & $\begin{array}{l}\text { O teor de iodo salino variou entre } 24,55 \\
\text { e } 39,6 \text { ppm. A prevalência de bócio foi de } \\
44,36 \% \text { nos escolares. } 37,8 \% \text { estavam com } \\
\text { deficiência de iodo leve, } 17,07 \% \text { moderada } \\
\text { e 3,05\% grave. } 32,43 \% \text { estavam com o T4 } \\
\text { abaixo dos valores normais para a idade e } \\
12,4 \% \text { com a Tg acima do normal. }\end{array}$ & $\begin{array}{l}\text { A palpação da tireoide está associada } \\
\text { à ocorrência de falsos positivos. } \\
\text { Já a ultrassonografia da tireoide é } \\
\text { confiável e precisa para diagnosticar } \\
\text { o bócio. A Tg reflete a nutrição } \\
\text { iódica por um período de meses ou } \\
\text { anos. }\end{array}$ \\
\hline $\begin{array}{l}\text { Zoysa, } \\
\text { Hettiarachchi, } \\
\text { Liyanage, } 2016^{4}\end{array}$ & WHO $(2007)^{1}$ & $\begin{array}{l}\text { A CUI foi de } 170,9 \mu \mathrm{g} / \mathrm{l} \text { no primeiro } \\
\text { trimestre, } 123,80 \mu \mathrm{g} / \mathrm{l} \text { no segundo e } 105,95 \\
\mu \mathrm{g} / \mathrm{l} \text { no terceiro. } 16 \% \text { tinham bócios } \\
\text { palpáveis e } 13,1 \% \text { tinham bócios palpáveis } \\
\text { mas não visíveis. O volume da tireoide foi } \\
\text { de } 5,16 \text { ml. O nível sérico de TSH foi } 1,3 \\
\mu \mathrm{IU} / \mathrm{ml} \text { no } 1^{\circ} \text { no trimestre e } 1,6 \mu \mathrm{IU} / \mathrm{ml} \text { no } \\
3^{\circ} \text { trimestre. }\end{array}$ & $\begin{array}{l}\text { A deficiência de iodo não foi } \\
\text { refletida pelo nível sérico de TSH, } \\
\text { pois o estado nutricional de iodo foi } \\
\text { piorando progressivamente e o TSH } \\
\text { manteve-se normal. }\end{array}$ \\
\hline
\end{tabular}

(I/24h) = iodúria de 24 horas; (I/Cr) = relação iodo/creatinina; (I/kg) = relação iodo por quilo corporal; (Tg) tiroglobulina; (CUI): concentração urinária de iodo; (TSH) hormônio estimulante da tireoide; (T3) triidotironina; (T4) tiroxina; (FT4) tiroxina livre; (WHO): Organização Mundial de Saúde; (UNICEF): Fundo das Nações Unidas para a Infância; (ICCIDD): Conselho Internacional para o Controle de Transtornos por Deficiência de Iodo; $(\mathrm{CV})$ = variação intra-individual; (UIE) = excreção urinária de iodo; (UIC): concentração urinária de iodo; (UI/Cr) iodúria/creatinina.

Fonte: Elaborado pelos autores.

para iodo sérico. Logo, para diagnóstico de deficiência de iodo, indica-se UI/Cr, e para excesso de iodo, recomenda-se o iodo sérico.

Outro indicador de impacto foi o volume tireoidiano, utilizado em $25 \%$ dos estudos. Ele pode ser verificado por meio de palpação ou ul- trassonografia, para determinar a prevalência de bócio. Em estudo realizado em 2002 na Nova Zelândia com escolares, os autores concluíram que o tamanho da tireoide das crianças pode variar de acordo com sexo, idade, crescimento, estado nutricional de iodo e herança genética, e que a li- 
Quadro 3. Pontos de corte para concentração urinária de iodo conforme o ano de referência.

\begin{tabular}{|c|c|c|c|c|}
\hline $\begin{array}{l}\text { Concentração } \\
\text { urinária de } \\
\text { iodo } \\
\end{array}$ & $\begin{array}{l}\text { WHO, UNICEF, } \\
\text { ICIDD (1992) }\end{array}$ & $\begin{array}{l}\text { WHO, UNICEF, } \\
\text { ICIDD (1994) }\end{array}$ & $\begin{array}{l}\text { WHO, UNICEF, ICIDD } \\
(2001)\end{array}$ & WHO, UNICEF, ICIDD (2007) \\
\hline $\begin{array}{l}\text { Todas as } \\
\text { populações }\end{array}$ & $\begin{array}{l}<20 \mu \mathrm{g} / \mathrm{l} \text { (grave); } \\
20-49 \mu \mathrm{g} / \mathrm{l} \\
\text { (moderado); } \\
50-99 \mu \mathrm{g} / \mathrm{l} \\
(\text { leve); } \geq 100 \mu \mathrm{g} / \mathrm{l} \\
\text { (adequado) }\end{array}$ & $\begin{array}{l}<20 \mu \mathrm{g} / \mathrm{L}(\text { grave)} ; \\
20-49 \mu \mathrm{g} / 1 \\
(\text { moderado); } \\
50-99 \mu \mathrm{g} / \mathrm{l} \\
(\text { leve) } \geq 100 \mu \mathrm{g} / \mathrm{l} \\
\text { (adequado) }\end{array}$ & $\begin{array}{l}<20 \mu \mathrm{g} / \mathrm{l} \text { (grave); } 20- \\
49 \mu \mathrm{g} / \mathrm{l}(\text { moderado); } 50- \\
99 \mu \mathrm{g} / \mathrm{l}(\text { leve); } 100-199 \mu \mathrm{g} / \mathrm{l} \\
\text { (adequado); } 200-299 \mu \mathrm{g} / \mathrm{l} \\
\text { (acima da recomendação); } \\
\geq 300 \mu \mathrm{g} / \mathrm{l} \text { (excesso) }\end{array}$ & 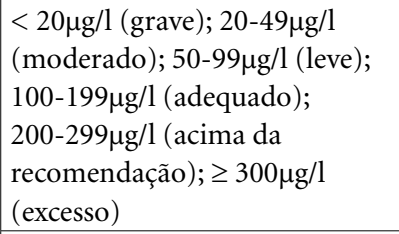 \\
\hline Gestantes & - & - & - & $\begin{array}{l}<150 \mu \mathrm{g} / \mathrm{l} \text { (insuficiente); } 150- \\
249 \mu \mathrm{g} / \mathrm{l} \text { (adequado); } 250-499 \mu \mathrm{g} / \\
\mathrm{l} \text { (acima da recomendação); } \geq \\
500 \mu \mathrm{g} / \mathrm{l} \text { (excesso) }\end{array}$ \\
\hline
\end{tabular}

(WHO): Organização Mundial de Saúde; (UNICEF): Fundo das Naçoes Unidas para a Infância; (ICCIDD): Conselho Internacional para o Controle de Transtornos por Deficiência de Iodo.

Fonte: Elaborado pelos autores.

Tabela 1. Percentual de acertos para os critérios da escala adaptada de Downs e Black ${ }^{8}$.

\begin{tabular}{|c|c|}
\hline Critérios & $\begin{array}{c}\text { Acertos } \\
(\%)\end{array}$ \\
\hline \multicolumn{2}{|l|}{ Relato de estudo } \\
\hline Objetivos/hipótese claramente descritos & $95 \%$ \\
\hline $\begin{array}{l}\text { Desfechos principais medidos e } \\
\text { descritos na metodologia }\end{array}$ & $100 \%$ \\
\hline $\begin{array}{l}\text { Definição dos critérios de inclusão e } \\
\text { exclusão }\end{array}$ & $75 \%$ \\
\hline Principais achados descritos & $100 \%$ \\
\hline $\begin{array}{l}\text { Variabilidade randômica dos dados } \\
\text { para os desfechos }\end{array}$ & $100 \%$ \\
\hline Perda de acompanhamento descrita & $60 \%$ \\
\hline $\begin{array}{l}\text { Valores reais de probabilidade } \\
\text { reportados integralmente }\end{array}$ & $100 \%$ \\
\hline \multicolumn{2}{|l|}{ Validade externa } \\
\hline Representatividade da amostra & $100 \%$ \\
\hline Análise não planejada reportada & $70 \%$ \\
\hline $\begin{array}{l}\text { Tempo de seguimento iguais para toda } \\
\text { a amostra }\end{array}$ & $100 \%$ \\
\hline Testes estatísticos apropriados & $95 \%$ \\
\hline $\begin{array}{l}\text { Medidas de desfecho válidas e } \\
\text { confiáveis }\end{array}$ & $100 \%$ \\
\hline \multicolumn{2}{|l|}{ Validade interna } \\
\hline $\begin{array}{l}\text { Indivíduos recrutados na mesma } \\
\text { população }\end{array}$ & $100 \%$ \\
\hline $\begin{array}{l}\text { Indivíduos recrutados no mesmo } \\
\text { período de tempo }\end{array}$ & $100 \%$ \\
\hline $\begin{array}{l}\text { Ajuste adequado para fatores de } \\
\text { confusão na análise }\end{array}$ & $80 \%$ \\
\hline Perdas de indivíduos reportados & $60 \%$ \\
\hline \multicolumn{2}{|l|}{ Poder do estudo } \\
\hline Apresenta o poder do estudo & $0 \%$ \\
\hline
\end{tabular}

Fonte: Elaborado pelos autores. mitação para utilizar a ultrassonografia para medir a tireoide infantil se devia a não haver pontos de corte para a classificação ${ }^{16}$. Entretanto, em estudo realizado com escolares no Egito, os autores destacaram que a ultrassonografia é mais confiável e precisa para diagnosticar o bócio, quando comparada à palpação da tireoide, que está associada à ocorrência de falsos positivos ${ }^{2}$.

Já em estudo realizado com gestantes, os autores destacaram que o aumento no volume da tireoide é um indicador indireto de deficiência de iodo e que mudanças na prevalência de bócio estão aquém de modificações no estado nutricional de iodo, por isso não refletem com precisão a ingestão atual de iodo ${ }^{23}$.

Também são utilizados para avaliar a função tireoidiana o hormônio estimulante da tireoide (TSH), a tireoglobulina (Tg), a triiodotironina (T3), a tiroxina (T4) e a tiroxina livre (FT4).

O TSH foi avaliado em $45 \%$ dos artigos. Os estudos que foram realizados com recém-nascidos concluíram que ele foi sensível para a vigilância da deficiência de iodo, sendo um bom indicador para monitorar o estado nutricional de iodo em nível populacional e individual ${ }^{14,21,22}$. O mesmo foi observado em estudo realizado com adultos, em que a mediana do TSH foi considerada um bom indicador para o monitoramento do estado nutricional da população com excesso e deficiência de iodo ${ }^{24}$.

Porém, em estudo realizado com gestantes, os autores concluíram que a deficiência de iodo não foi refletida pelo nível sérico de TSH, pois o estado nutricional de iodo foi se agravando progressivamente com a gestação (CUI de 170,9 $\mu \mathrm{g} / \mathrm{l}$ no primeiro trimestre, $123,80 \mu \mathrm{g} / \mathrm{l}$ no segundo e 
$105,95 \mu \mathrm{g} / \mathrm{l}$ no terceiro) mas mesmo assim o TSH se manteve adequado ${ }^{4}$. Em outro estudo com gestantes, a $\mathrm{Tg}$ foi considerada um indicador mais sensível para avaliar a deficiência de iodo durante a gestação quando comparada ao $\mathrm{TSH}^{13}$. No entanto, Soldin e colaboradores ${ }^{18}$ observaram que T3, FT4, TSH e Tg tendem a mudar ao longo da gestação, por isso não são bons indicadores para avaliar a deficiência de iodo.

Dessa forma, é preciso que mais estudos sejam realizados para determinar qual o melhor indicador para avaliar o estado nutricional de iodo na gestação, a fim de contornar as divergências que até hoje são detectadas.

A Tg foi avaliada em $20 \%$ dos artigos. Em estudo realizado com escolares, o percentil de altura e a Tg foram indicadores sensíveis para demonstrar o efeito crônico da deficiência de iodo, pois refletiram o estado nutricional pregresso e foram mais eficazes do que TSH e T4 para classificar a gravidade da deficiência de iodo ${ }^{17}$. No estudo de Yamamah e colaboradores ${ }^{2}$ com escolares, a $\mathrm{Tg}$ refletiu a nutrição iódica por um período de meses ou anos, por isso foi considerada um bom indicador para avaliar o estado nutricional de iodo. Já em estudo realizado com indivíduos de 15 a 44 anos, T4 e TSH foram considerados indicadores indiretos da deficiência de iodo ${ }^{19}$.

Um indicador que não é descrito pela $\mathrm{OMS}^{1}$, mas que foi estudado por Prejac e colaboradores ${ }^{5}$ em 2014, é a taxa de iodo nos fios de cabelo, que reflete a ingestão dietética. Os autores observaram que a mediana da concentração de iodo no cabelo entre homens e mulheres adultos foi de $0.499 \mu \mathrm{gg}^{-1}$, sugerindo inadequação na ingestão de iodo. O cabelo registra uma série de eventos metabólicos intermediários e o controle homeostático de todos os elementos essenciais, entre eles a concentração de iodo sanguíneo, por isso é um indicador valioso e robusto para a avaliação da ingestão dietética de iodo individual em longo prazo. Entretanto, conforme o dia de avaliação, a concentração pode estar alterada.

TSH, Tg, T3, T4 e FT4 são indicadores diretos do funcionamento da tireoide, e indiretos do estado nutricional de iodo. O TSH em recémnascidos foi mais sensível para diagnóstico da deficiência de iodo, embora os estudos tenham relatado dificuldades na interpretação dos resultados. E a Tg foi mais sensível para a avaliação do estado nutricional de iodo em gestantes e escolares. Já a presença de iodo no cabelo mostrou-se um indicador útil para avaliação do estado nutricional de adultos.

\section{Indicadores de sustentabilidade}

Determinam se a deficiência de iodo foi erradicada com sucesso e avaliam se as conquistas podem ser sustentadas pelas próximas décadas. Para isso, utiliza-se a CUI populacional e a disponibilidade de sal iodado no domicílio, em conjunto com os outros indicadores de sustentabilidade ${ }^{1}$.

E, 2008, Ategbo e colaboradores ${ }^{20}$ avaliaram o consumo de sal iodado em 1.200 domicílios e observaram que o teor de iodo salino foi adequado ( $\geq 15$ ppm) em 41,9\% deles, porém nos domicílios que não utilizavam sal iodado a mediana da CUI foi menor em gestantes e crianças.

É notável o progresso dos programas para controle da deficiência de iodo nas últimas décadas, porém os DDI podem retornar a qualquer momento se essas estratégias não forem sustentadas $^{1}$.

Em estudo realizado com escolares na Etiópia, a prevalência de bócio foi maior (53,3\%) nas crianças que viviam nas aldeias em que a água tinha baixa concentração de iodo e era contaminada com coliformes. Observou-se ainda que o teor de iodo nos alimentos variou de acordo com o local em que eram produzidos, o que já era esperado, segundo os autores, pois o teor de iodo nos alimentos depende de sua concentração no solo $^{12}$. E no estudo realizado com adultos, os autores observaram que a mediana da concentração de iodo na água potável foi menor no grupo deficiente em iodo $(7,69 \mathrm{mg} / \mathrm{l}$ a $77,77 \mathrm{mg} / \mathrm{l})$ e maior no grupo com excesso $(272,98 \mathrm{mg} / \mathrm{l} \mathrm{a}$ $629,90 \mathrm{mg} / \mathrm{l})^{24}$.

\section{Recomendações gerais na avaliação do estado nutricional de iodo}

Segundo a OMS, deve-se utilizar a CUI para determinar o estado nutricional de iodo e o impacto da iodação do sal na população. Para que a análise da manutenção do programa de iodação seja realizada periodicamente, é preciso apoio político, de forma que o programa seja regulamentado e tenha recursos para realizar suas atividades. E cabe ao estado garantir a qualidade, reduzir os custos do sal e solicitar relatórios periódicos para determinar o estado de iodo na população ${ }^{1}$.

Porém, a realidade é que mesmo onde os programas de iodação do sal já possuem alta cobertura, ainda existe vulnerabilidades referentes a apoio político, recurso financeiro, conscientização e aceitação do consumidor, o que dificulta 
a manutenção da iodação universal do sal pelas próximas décadas.

Para avaliar o volume tireoidiano com vistas a analisar a nutrição iódica, a OMS não recomenda a utilização da palpação, uma vez que essa tem baixa sensibilidade para as mudanças agudas na ingestão de iodo e a classificação pode variar entre os observadores. No entanto, pode ser útil para avaliar a gravidade e o impacto dos programas de controle dos DDI em longo prazo. Já a ultrassonografia da tireoide é utilizada para a triagem de bócio, por isso é o indicador normalmente utilizado em áreas já diagnosticadas com deficiência de iodo leve a moderada ${ }^{1}$.

\section{Pontos fortes e limitações}

Como pontos fortes dessa revisão, destacamos que todos os biomarcadores do estado nutricional de iodo foram contemplados, bem como todas as faixas etárias. A limitação é que, como a busca foi feita sem delimitação de datas, foram incluídos estudos realizados há cerca de 20 anos e com referências antigas para a classi-

\section{Colaboradores}

AC Candido foi responsável pela concepção e desenho do estudo e pela interpretação dos dados e redação do artigo. FM Azevedo foi responsável pela interpretação dos dados e a revisão crítica do artigo. MS Macedo, SE Priore e SCC Franceschini orientaram o trabalho e realizaram a revisão crítica do artigo. Todas as autoras aprovaram a versão a ser publicada.

\section{Agradecimentos}

O presente trabalho foi realizado com apoio da Coordenação de Aperfeiçoamento de Pessoal de Nível Superior (Capes), do Conselho Nacional de Desenvolvimento Científico e Tecnológico (CNPq) e da Fundação de Amparo à Pesquisa do Estado de Minas Gerais (Fapemig). ficação da concentração urinária de iodo. No entanto, esses estudos foram úteis para confrontar o uso de diferentes metodologias e indicadores para diagnóstico do estado nutricional de iodo no decorrer dos anos.

\section{Conclusão}

É necessário cautela na escolha do biomarcador para analisar o estado nutricional de iodo. Primeiro, é essencial definir se a avaliação será individual ou populacional, depois é preciso levar em consideração a faixa etária e o estado fisiológico.

De acordo com essa revisão, recomenda-se a concentração urinária de iodo (CUI) para diagnóstico de deficiência e excesso de iodo na população. Para a avaliação individual do estado nutricional de iodo, indica-se o hormônio estimulante da tireoide (TSH) em recém-nascidos, o iodo no cabelo em adultos e a tiroglobulina ( $\mathrm{Tg}$ ) em escolares e gestantes. E para o diagnóstico de excesso de iodo em gestantes e puérperas, o iodo sérico foi considerado um bom biomarcador.

\section{Referências}

1. World Health Organization (WHO). Assessment of the iodine deficiency disorders and monitoring their elimination. Geneva: WHO; 2007.

2. Yamamah GA, Salah MM, Hussieny MS, Ibrahim MH Monitoring of iodine deficiency disorders for South Sinai children (IDD monitoring in South Sinai). Int $J$ Pharm Clin Res 2016; 8(8):112711-31.

3. Zimmermann M. Iodine deficiency and excess in children: worldwide status in 2013. Endocr Pract 2013; 19(5):839-846.

4. Zoysa E, Hettiarachchi M, Liyanage C. Urinary iodine and thyroid determinants in pregnancy: a follow up study in Sri Lanka. BMC Pregnancy Childbirth 2016; 16(1):1-6.

5. Prejac J, Visnjevic V, Drmic S, Skalny AA, Mimica N, Momcilovic B. A novel concept to derive iodine status of human populations from frequency distribution properties of a hair iodine concentration. J Trace Elem Med Biol 2014; 28(2):205-211.

6. Li C, Peng S, Zhang X, Xie X, Wang D, Mao J, Teng X, Shan $Z$, Teng $W$. The urine iodine to creatinine as an optimal index of iodine during pregnancy in an iodine adequate area in China. J Clin Endocrinol Metab 2016; 101(3):1290-1298. 
7. Liberati A, Altman DG, Tetzlaff J, Mulrow C, Gotzsche PC, Ioannidis JPA, Clarke M, Devereaux PJ, Kleijnen J, Moher D. The PRISMA statement for reporting systematic reviews and meta-analyses of studies that evaluate health care interventions: explanation and elaboration. PLoS Med 2009; 339:b2700.

8. Downs S, Black N. The feasibility of creating a checklist for the assessment of the methodological quality of health care interventions. J Epidemiol Community Heal 1998; 52:377-384.

9. Brug J, Lowik MRH, Van Binsbergen JJ, Odink J, Egger RJ, Wedel M. Indicators of iodine status among adults: Dutch nutrition surveillance system. Ann Nutr Metab 1992; 36(3):129-134.

10. Konig F, Andersson M, Hotz K, Aeberli I, Zimmermann MB. Ten repeat collections for urinary iodine from spot samples or 24-hour samples are needed to reliably estimate individual iodine status in women. $J$ Nutr 2011; 141(11):2049-2054.

11. Santiago MI, Fernández S, Rios M, Fluiters E, Hervada $\mathrm{X}$, Iglesias T. Excreción urinaria de yodo en escolares de Galicia. Endocrinol y Nutr 2005; 52(9):498-505.

12. Cherinet A, Kelbessa U. Determinants of iodine deficiency in school children in different regions of Ethiopia. East Afr Med J 2000; 77(3):133-137.

13. Eltom A, Elnagar B, Elbagir M, Gebre-Medhin M. Thyroglobulin in serum as an indicator of iodine status during pregnancy. Scand J Clin Lab Invest 2000; 60(1):1-7.

14. Costante G, Grasso L, Schifino E, Marasco MF, Crocetti U, Capula C, Chiarella R, Ludovico O, Nocera M, Parlato G, Filetti S. Iodine deficiency in Calabria: characterization of endemic goiter and analysis of different indicators of iodine status region-wide. J Endocrinol Invest 2002; 25(3):201-207.

15. Delange F, Benoist B, Burgi H, ICCIDD Working Group. Determining median urinary iodine concentration that indicates adequate iodine intake at population level. Bull World Health Organ 2002; 80(8):633636.

16. Skeaff SA, Thomson CD, Gibson RS. Mild iodine deficiency in a sample of New Zealand schoolchildren. Eur J Clin Nutr 2002; 56(12):1169-1175.

17. Simsek E, Safak A, Yavuz O, Aras S, Dogan S, Kocabay K. Sensitivity of iodine deficiency indicators and iodine status in Turkey. J Pediatr Endocrinol Metab 2003; 16(2):197-202.

18. Soldin OP, Tractenberg RE, Hollowell JG, Jonklaas J, Janicic N, Soldin SJ. Trimester-specific changes in maternal thyroid hormone, thyrotropin, and thyroglobulin concentrations during gestation: trends and associations across trimesters in iodine sufficiency. Thyroid 2004; 14(12):1084-1090.

19. Soldin OP, Tractenberg RE, Pezzullo JC. Do thyroxine and thyroid-stimulating hormone levels reflect urinary iodine concentrations? Ther Drug Monit 2005; 27(2):178-185.

20. Ategbo EA, Sankar R, Schultink W, Haar F, Pandav CS. An assessment of progress toward universal salt iodization in Rajasthan, India, using iodine nutrition indicators in school-aged children and pregnant women from the same households. Asia Pac J Clin Nutr 2008; 17(1):56-62.
21. Dorey CM, Zimmermann MB. Reference values for spot urinary iodine concentrations in iodine-sufficient newborns using a new pad collection method. Thyroid 2008; 18(3):347-352.

22. Barona-Vilar C, Fullana-Montoro RMA, Barona-Vilar C. Neonatal thyrotropinemia (TSH) as an indicator of iodine nutritional level in Castellon and Valencia, Spain (2004-2006). Rev Esp Salud Publica 2008; 82(4):405-413.

23. Elahi S, Rizvi NB, Nagra SA. Iodine deficiency in pregnant women of Lahore. J Pak Med Assoc 2009; 59(11):741-743.

24. Du Y, Gao Y, Meng F, Liu S, Fan Z, Wu J, Sun D. Iodine deficiency and excess coexist in China and induce thyroid dysfunction and disease: a cross-sectional study. PLoS One 2014; 9(11):e111937.

25. Hetzel BS, Dunn JT. The iodine deficiency disorders: their nature and prevention. Annu Rev Nutr 1989; 9(1):21-38.

26. World Health Organization (WHO), International Council for Control of Iodine Deficiency, United Nations Children's Fund (UNICEF). Indicators for assessing iodine deficiency disorders and their control through salt iodization. WHO; 1994.

27. Disorders D. Recommended normative values for thyroid volume in children aged 6-15 years. Bull World Health Organ 1997; 75(2):95-97.

28. Soukhova N, Soldin OP, Soldin SJ. Isotope dilution tandem mass spectrometric method for T4/T3. Clin Chim Acta 2004; 343(1-2):185-190.

29. Rartels H, Bohmer M. Eine mikromethode 7air kreatininbestimmung. Clin Chim Acta 1971; 32(1):81-85.

30. Pino S, Fang SL, Braverman LE. Ammonium persulfate: a safe alternative oxidizing reagent for measuring urinary iodine. Clin Chem 1996; 42(2):239-243.

31. Euser AM, Dekker FW, Cessie S. A practical approach to Bland-Altman plots and variation coefficients for log transformed variables. J Clin Epidemiol 2008;v61(10):978-982.

32. Yengar GV, Sansoni B. Elemental Analysis of Biological Materials: Current Problems and Techniques with Special Reference to Trace. Viena: IAEA;1980. (Elements Technical Report S Series, N.197).

Artigo apresentado em 29/10/2018

Aprovado em 30/09/2019

Versão final apresentada em 02/10/2019

Editores-chefes: Romeu Gomes, Antônio Augusto Moura da Silva 